Artigo / Article

\title{
Marcadores de prognóstico na leucemia linfocítica crônica
}

\section{Prognostic factors in chronic lymphocytic leukemia}

Yuri Vasconcelos

\begin{abstract}
A leucemia linfocítica crônica $(L L C)$ é reconhecida pela evolução clínica heterogênea que não consegue ser prevista com exatidão pelos sistemas de estadiamento clínico a nivel individual. Isto levou à investigação de outros marcadores de prognóstico que poderiam agregar valor preditivo aos sistemas de estadiamento ou até mesmo substitui-los. Entre os marcadores clínicos e biológicos inicialmente encontrados, as aberrações cromossômicas e o estado mutacional dos genes de imunoglobulinas demonstraram uma alta precisão na avaliação de prognóstico na LLC. No entanto, as técnicas empregadas nestes estudos são laboriosas e inacessiveis à maioria dos serviços de onco-hematologia, o que motivou a busca por marcadores substitutos (surrogate). Entre os potenciais marcadores surrogate, CD38 e Zap-70 possuem um papel independente de prognóstico na $L L C$, com um poder de predição evolutiva tão (ou mais) preciso quanto o perfil mutacional das imunoglobulinas, possibilitando sua substituição definitiva num futuro próximo. Novos marcadores como LPL, LPL/ADAM29 e Vimentina têm apresentado resultados preliminares bastante atrativos, porém ainda aguardam validação em outras séries de pacientes. Mesmo com a identificação de marcadores biológicos altamente precisos, os sistemas de estadiamento clínico ainda não devem ser abandonados. Rev. bras. hematol. hemoter. 2005;27(4):253-256.
\end{abstract}

Palavras-chave: Leucemia linfocítica crônica; prognóstico.

\section{Introdução}

A leucemia linfocítica crônica (LLC) é o tipo mais freqüente de leucemia em adultos nos países ocidentais, e é caracterizada por uma evolução clínica bastante heterogênea entre os indivíduos acometidos, com sobrevida que varia de alguns meses até algumas décadas. ${ }^{1}$ Cerca de $70 \%$ dos pacientes apresentam-se assintomáticos ao diagnóstico, e mais da metade destes não irá apresentar quaisquer eventos secundários à doença hematológica. Por outro lado, os demais indivíduos costumam apresentar progressão da doença, demanda terapêutica e causas de morte relacionadas à LLC. ${ }^{2}$

Há quase trinta anos, os sistemas de estadiamento clínico desenvolvidos por Rai e Binet estratificaram os portadores de LLC em três grupos de risco: baixo (Rai 0, Binet A), intermediário (Rai I/II, Binet B) e alto (Rai III/IV, Binet C), apresentando uma mediana de sobrevida estimada em
$>10$ anos, 5-7 anos e 1-3 anos, respectivamente. ${ }^{3,4,5}$ Entretanto, ainda que tenham servido de um bom parâmetro para decisão terapêutica na prática médica, nenhum destes sistemas é capaz de aferir precisamente o prognóstico de um paciente com LLC. ${ }^{6}$ Esta limitação inerente aos sistemas de estadiamento clínico deu início a uma busca por marcadores clínicos e biológicos que pudessem aprimorar a predição evolutiva dos portadores de LLC. Alguns destes marcadores acabaram se revelando simples tradutores da carga tumoral, enquanto outros puderam desvendar alguns aspectos relevantes da patologia molecular na LLC.

\section{Marcadores clínicos de prognóstico na LLC}

\section{Sistemas de estadiamento clínico}

Os pacientes com LLC são mundialmente alocados em três grandes grupos de risco (baixo, intermediário e alto)

Instituto Goiano de Oncologia e Hematologia - INGOH.

Correspondência para: Yuri Vasconcelos

Instituto Goiano de Oncologia e Hematologia - INGOH

Rua 87, 598 - Setor Sul

74093-300 - Goiânia-GO - Brasil

Tel.: (62) 32260200 -Fax.: (62) $32815301-E$-mail: yvasconc@ingoh.com.br 
baseando-se em dois sistemas de estadiamento clínico, seja o de Rai e/ou de Binet. ${ }^{3,4}$ Nos países da América do Norte, utiliza-se mais freqüentemente o primeiro, ao passo que o segundo é mais empregado nos países europeus. No Brasil, a preferência entre Rai e Binet varia entre os serviços de onco-hematologia. Há inclusive centros que lançam mão das duas classificações (ex.: Rai 0, Binet A). Aqui, como em vários países, não existe um consenso de qual deles poderia ser superior na avaliação de prognóstico na LLC.

Do ponto de vista prático, Binet parece ser mais objetivo que Rai na categorização dos portadores de LLC, pois os indivíduos Binet A correspondem ao dobro dos classificados em Rai 0 (63\% vs. $31 \%$ dos casos de LLC, respectivamente), no entanto possuindo uma mediana de sobrevida equivalente ( $>10$ anos para ambos). ${ }^{5}$ Infelizmente, apesar da fácil aplicação de um ou outro sistema de estadiamento na prática clínica (ambos dependem apenas de exame físico e hemograma), nenhum destes consegue prever as variações evolutivas individuais e acabam por somente refletir a carga tumoral.

\section{Idade, raça e sexo}

Nem idade nem raça possuem um papel relevante na avaliação de prognóstico na LLC. Pacientes idosos costumam evoluir menos favoravelmente em função de suas comorbidades. ${ }^{7}$ Apesar de raramente encontrada em populações do extremo oriente, o prognóstico dos indivíduos acometidos por LLC não difere daqueles oriundos de outras raças. ${ }^{8}$ Por outro lado, além de uma incidência ligeiramente maior de pessoas do sexo masculino entre os casos de LLC, estes geralmente evoluem de forma mais desfavorável quando comparados às pacientes do sexo feminino. ${ }^{9} \mathrm{O}$ motivo desta distinção evolutiva ainda permanece especulativo.

\section{Marcadores biológicos de prognóstico na LLC}

\section{Tempo de duplicação linfocitária}

Um tempo de duplicação linfocitária $\leq 12$ meses confere um pior prognóstico, ${ }^{10} \mathrm{e}$ muitas vezes serve de parâmetro (se $<6$ meses) na decisão terapêutica de pacientes com LLC. ${ }^{11}$ Apesar de classicamente utilizado, o tempo de duplicação linfocitária vem perdendo importância na estimativa de prognóstico na LLC, tanto pela longa espera na sua definição quanto pelo poder preditivo superior de outros parâmetros biológicos (imunofenotípicos e moleculares) que serão discutidos mais adiante.

Padrão de infiltração na histologia da medula óssea

Os portadores de LLC contendo um padrão difuso de infiltração linfocitária na histologia da medula óssea possuem historicamente um perfil evolutivo mais agressivo quando comparados aos pacientes que apresentam um padrão não-difuso. ${ }^{12}$ Como a biópsia de medula óssea está atualmente reservada a raros casos em que auxilia no diagnóstico diferencial com outras doenças linfoproliferativas crô- nicas, o padrão histomorfológico da medula óssea encontra-se em desuso na análise de prognóstico na LLC.

\section{Marcadores séricos}

Os altos níveis séricos de beta2-microglobulina, desidrogenase lática, timidina quinase e CD23 solúvel correlacionam com uma sobrevida inferior em indivíduos com LLC, porém existe grande variação nos pontos de corte e na metodologia empregada entre as séries publicadas. ${ }^{13-17}$ Além disso, estes estudos basearam-se exclusivamente em análises retrospectivas, sem uma seleção criteriosa dos pacientes envolvidos. Portanto, apesar de serem acessíveis à maioria dos serviços de onco-hematologia, o uso destes marcadores não parece contribuir de forma significativa à avaliação de prognóstico na LLC.

\section{Imunofenótipo}

O estudo do imunofenótipo celular é indispensável ao diagnóstico de LLC, ${ }^{18}$ e também serve como importante ferramenta na apreciação de três marcadores de prognóstico nesta doença: CD38, Zap-70 e lipoproteína lipase (LPL). Seus altos níveis de expressão nos linfócitos B da LLC estão freqüentemente associados a pacientes com prognóstico desfavorável, independentemente do estádio clínico, ${ }^{19-26}$ o que parece correlacionar com um maior estado de ativação celular. ${ }^{27-30} \mathrm{~A}$ metodologia mais comumente empregada na avaliação destes marcadores é a citometria de fluxo multiparamétrica. Enquanto CD38 e Zap-70 possuem anticorpos comercialmente disponíveis, a LPL ainda carece desta disponibilidade. Os estudos realizados com CD38 e com Zap-70 oferecem dados contraditórios em relação ao valor de expressão utilizado como ponto de corte, porém o importante é considerar a porcentagem de linfócitos B com alta expressão destes marcadores, já que subpopulações celulares - contendo padrões distintos de expressão - podem coexistir numa mesma amostra analisada. De maneira geral, os pontos de corte mais amplamente utilizados são $30 \%$ e $20 \%$ para CD38 e Zap-70, respectivamente. ${ }^{19,22}$ Para LPL, o valor ideal ainda requer validação em séries com maior número de pacientes. ${ }^{26}$

\section{Aberrações cromossômicas}

A LLC, ao contrário de outras doenças linfoproliferativas crônicas, não possui nenhuma alteração citogenética característica. Entre as aberrações genéticas mais conhecidas, algumas figuram como indicadores de bom prognóstico (13q- isolada) e outras como marcadores de risco intermediário (cariótipo normal, $+12 \mathrm{q}$ ) ou alto $(11 \mathrm{q}-, 17 \mathrm{p}-$ ). A deleção $17 \mathrm{p}$ confere um prognóstico especialmente reservado ao paciente com LLC, notadamente pela má resposta a tratamentos convencionais com agentes alquilantes e análogos de purina, e por uma mediana de sobrevida inferior a três anos. ${ }^{31-32}$ As técnicas normalmente empregadas na avaliação citogenética de portadores de LLC são as de bandeamento (cariótipo) e FISH. Apesar de uma visão mais ampla do perfil cromossômico, a técnica de bandeamento pode apresentar dificuldades em sua execução (os linfócitos B da 
LLC possuem baixa taxa mitótica) e resultados falso-negativos (ex.: uma pequena deleção pode passar despercebida e ser interpretada como cariótipo normal). Quanto ao FISH, o fator limitante é o custo, pois para uma análise precisa, seria necessário um painel mínimo com quatro sondas (para $11 \mathrm{q}-,+12,13 \mathrm{q}-\mathrm{e} 17 \mathrm{p}-)$.

\section{Estado de mutação dos genes de imunoglobulinas}

Nos últimos dez anos, alguns estudos constataram que o estado de mutação dos genes da região variável da cadeia pesada de imunoglobulinas (genes $I g V H$ ) confere uma predição evolutiva bastante acurada aos pacientes com LLC. De forma comparativa à seqüência germline de referência, indivíduos com uma homologia $<98 \%$ costumam apresentar uma evolução clínica bastante indolente e mediana de sobrevida que excede os vinte anos. Por outro lado, os pacientes com uma homologia $\geq 98 \%$ freqüentemente possuem doença de caráter agressivo, demanda terapêutica, alta incidência de morte por causas relacionadas à LLC, e uma mediana de sobrevida em torno de oito anos. ${ }^{19,32,33}$

Desde então, os portadores de LLC passaram a ser categorizados em "mutados" e "não-mutados", com o perfil mutacional $\operatorname{IgVH}$ passando a ser considerado como padrãoouro na avaliação de prognóstico na LLC. No entanto, a metodologia empregada é bastante laboriosa e pouco aplicável na prática rotineira dos serviços de onco-hematologia. Por esta razão, marcadores de prognóstico que pudessem ser substitutos (surrogate) à análise $I g V H$ começaram a ser investigados. Entre os candidatos a surrogate, CD38, Zap70 , e LPL prevaleceram como os de maior potencial preditivo do perfil mutacional $\operatorname{IgVH}$, sendo que a alta expressão dos mesmos geralmente se correlaciona com casos não-mutados, e a baixa expressão com indivíduos mutados. ${ }^{19-26,34,35}$

\section{Novos marcadores de prognóstico na LLC}

Novos marcadores, como a razão de expressão $L P L /$ $A D A M 29^{25}$ e a Vimentina (Nowakowski et al. Leukemia Lymphoma 2005;46(suppl 1):S37; abstract), também possuem alta predição do estado mutacional $\operatorname{IgVH}$ e um valor independente de prognóstico na LLC, porém necessitam estudos confirmatórios.

\section{Discussão}

Os sistemas de estadiamento clínico de Rai e Binet são marcos na estratificação de risco dos pacientes com LLC., ${ }^{3,4}$ Embora tenham servido até os dias atuais na decisão terapêutica na prática médica, nenhum sistema consegue precisar o prognóstico individual de um paciente acometido pela doença. Tradicionalmente, os pacientes do grupo de baixo risco (Rai 0, Binet A) seguem uma conduta expectante ("esperar e ver") até apresentarem sinais de progressão da doença, enquanto os pacientes dos demais grupos de risco costumam ser sistematicamente tratados ao diagnóstico., ${ }^{5,11}$

Outros marcadores clínicos e biológicos começaram a ser investigados para agregar valor preditivo aos sistemas de estadiamento, ou mesmo substituí-los. Nos últimos dez anos, com o advento de novas modalidades de tratamento na LLC, esforços foram intensificados para a identificação precoce de marcadores adversos numa fase ainda "latente" da doença, e conseqüentemente com menor carga tumoral. Assim, os resultados terapêuticos podem parecer bem mais promissores do que os classicamente relatados com clorambucil.

Dos novos marcadores, os níveis séricos de beta2microglobulina, desidrogenase lática, timidina quinase e CD23 solúvel acabaram por se revelar em meros tradutores da carga tumoral. Por outro lado, o imunofenótipo celular, as aberrações cromossômicas e o estado mutacional $I g V H$ se estabeleceram como fatores independentes e de alto impacto na predição de prognóstico individual dos portadores de LLC.

Num passado mais recente, as dificuldades inerentes aos estudos citogenéticos e moleculares motivaram à busca de marcadores surrogate mais acessíveis à rotina dos serviços de onco-hematologia. Entre estes marcadores, sobressaem-se CD38, Zap-70 e LPL, todos passíveis de investigação por citometria de fluxo multiparamétrica, uma metodologia mais rápida e barata, mas que ainda carece de maior disponibilidade em nosso país.

Mesmo que algumas séries publicadas sugiram discordâncias da ordem de 10\%-30\% na correspondência entre as expressões de CD38 e Zap-70 com o status $I g V H$, estes marcadores persistem como variáveis independentes de prognóstico na LLC, com poder de predição evolutiva tão (ou mais) preciso quanto o perfil mutacional $I g V H$. Além do potencial de definitivamente substituir as análises de citogenética e de imunoglobulinas, CD38 e Zap-70 podem se constituir em alvos terapêuticos. Os resultados preliminares do perfil de expressão de LPL, LPL/ADAM29 e Vimentina também têm sido muito atraentes. Por outro lado, o padrão histomorfológico da medula óssea e o tempo de duplicação linfocitária poderão configurar em breve entre os anais históricos da LLC.

Finalmente vale ressaltar que, até o momento, mesmo com a identificação de marcadores biológicos altamente precisos, os sistemas de estadiamento clínico ainda possuem um papel independente de prognóstico na LLC, ${ }^{36}$ e que tampouco existem estudos prospectivos randomizados com resultados que já nos permitam tomar novos posicionamentos quanto ao manejo dos pacientes assintomáticos. Por enquanto, a aplicação prática de CD38 e Zap-70 deveria permanecer reservada a um contexto exclusivo de ensaios clínicos.

\footnotetext{
Abstract

Chronic lymphocytic leukemia is characterized by a variable clinical course that cannot be predicted accurately by clinical staging systems in individual patients. This prompted the investigation of other prognostic factors capable of adding predictive power to clinical staging systems or even substituting them. Among the clinical and biological markers found initially, genomic aberrations and
} 
the mutational status of immunoglobulin genes demonstrated a high level of prognostic prediction in CLL. However, the techniques employed in these studies are laborious and inaccessible for most hematology-oncology facilities, which motivated the hunt for surrogate markers. Among the potential surrogates, CD38 and Zap-70 play an independent prognostic role in CLL, with a predictive power as precise as (or even better than) the immunoglobulin mutational profile, pointing for its replacement in the near future. Novel factors such as LPL, LPL/ADAM29 and Vimentin have showed quite attractive preliminary results, but still wait for validation in further series of patients. Besides the recognition of powerful biological markers, the clinical staging systems should not be abandoned so far. Rev. bras. hematol. hemoter. 2005;27(4):253-256.

Key words: Chronic lymphocytic leukemia; prognosis.

\section{Referências Bibliográficas}

1. de Faria JR, de Oliveira JS, Delbone de Faria RM et al. Prognosis related to staging systems for chronic lymphocytic leukemia. Sao Paulo Med J 2000;118(4):83-88.

2. Dighiero G, Maloum K, Desablens B et al. Chlorambucil in indolent chronic lymphocytic leukemia. N Engl J Med 1998;338(21):1.506-1.514.

3. Rai KR, Sawitsky A, Cronkite EP et al. Clinical staging of chronic lymphocytic leukemia. Blood 1975;46(2):219-234.

4. Binet JL, Auquier A, Dighiero G et al. A new prognostic classification of chronic lymphocytic leukemia derived from a multivariate survival analysis. Cancer 1981;48(1):198-206.

5. Dighiero G, Binet JL. When and how to treat chronic lymphocytic leukemia. N Engl J Med 2000;343(24):1.799-1.801.

6. Keating MJ. Chronic lymphocytic leukemia. Semin Oncol 1999;26(5 Suppl 14):107-114.

7. Mauro FR, Foa R, Giannarelli D et al. Clinical characteristics and outcome of young chronic lymphocytic leukemia patients: a single institution study of 204 cases. Blood 1999;94:448-454.

8. Tamura K, Sawada H, Izumi Y et al. Chronic lymphocytic leukemia (CLL) is rare, but the proportion of T-CLL is high in Japan. Eur J Haematol 2001;67(3):152-157.

9. Catovsky D, Fooks J \& Richards S. Prognostic factors in chronic lymphocytic leukaemia: the importance of age, sex and response to treatment in survival. British Journal of Haematology 1989;72:141-149.

10. Montserrat E, Sanchez-Bisono J, Vinolas N et al. Lymphocyte doubling time in chronic lymphocytic leukaemia: analysis of its prognostic significance. Br J Haematol 1986;62:567.

11. Cheson BD, Bennett JM, Grever M et al. National Cancer Institute-sponsored Working Group guidelines for chronic lymphocytic leukemia: revised guidelines for diagnosis and treatment. Blood 1996;87(12):4.990-4.997.

12. Rozman C, Montserrat E, Rodriguez-Fernandez JM, et al. Bone marrow histologic pattern-the best single prognostic parameter in chronic lymphocytic leukemia: a multivariate survival analysis of 329 cases. Blood 1984;64:642.

13. Molica S, Levato D, Dell'Olio M et al. Cellular expression and serum circulating levels of CD23 in B-cell chronic lymphocytic leukemia. Implications for prognosis. Haematologica 1996;81(5):428-433.

14. Sarfati M, Chevret S, Chastang C et al. Prognostic importance of serum soluble CD23 level in chronic lymphocytic leukemia. Blood 1996; 88 (11): 4.259-4.264.

15. Molica S, Levato D, Cascavilla $\mathrm{N}$ et al. Clinico-prognostic implications of simultaneous increased serum levels of soluble $\mathrm{CD} 23$ and beta2-microglobulin in B-cell chronic lymphocytic leukemia. Eur J Haematol 1999;62(2):117-122.

16. Hallek M, Langenmayer I, Nerl C et al. Elevated serum thymidine kinase levels identify a subgroup at high risk of disease progression in early, nonsmoldering chronic lymphocytic leukemia. Blood 1999;93(5):1.732-1.737.
17. Magnac C, Porcher R, Davi F et al. Predictive value of serum thymidine kinase level for Ig-V mutational status in B-CLL. Leukemia 2003; 17(1):133-137.

18. Moreau EJ, Matutes E, A'Hern RP et al. Improvement of the chronic lymphocytic leukemia scoring system with the monoclonal antibody SN8 (CD79b). Am J Clin Pathol 1997;108(4):378-382.

19. Damle RN, Wasil T, Fais F et al. Ig V gene mutation status and CD38 expression as novel prognostic indicators in chronic lymphocytic leukemia. Blood 1999;94(6):1.840-1.847.

20. Ibrahim S, Keating M, Do KA et al. CD38 expression as an important prognostic factor in B-cell chronic lymphocytic leukemia. Blood 2001;98(1):181-186.

21. Del Poeta G, Maurillo L, Venditti A et al. Clinical significance of CD38 expression in chronic lymphocytic leukemia. Blood 2001;98(9):2.633-2.639.

22. Crespo M, Bosch F, Villamor N et al. ZAP-70 expression as a surrogate for immunoglobulin-variable-region mutations in chronic lymphocytic leukemia. N Engl J Med 2003;348(18):1.764-75.

23. Durig J, Nuckel H, Cremer M e al. ZAP-70 expression is a prognostic factor in chronic lymphocytic leukemia. Leukemia 2003; 17(12): 2.426-34.

24. Rassenti LZ, Huynh L, Toy TLet al. ZAP-70 compared with immunoglobulin heavy-chain gene mutation status as a predictor of disease progression in chronic lymphocytic leukemia. N Engl J Med 2004;351(9):893-901.

25. Oppezzo P, Vasconcelos Y, Settegrana C et al. The LPL/ADAM29 expression ratio is a novel prognosis indicator in chronic lymphocytic leukemia. Blood 2005;106(2):650-657.

26. Heintel D, Kienle D, Shehata M et al. High expression of lipoprotein lipase in poor risk B-cell chronic lymphocytic leukemia. Leukemia 2005; 19 : $1.216-1.223$

27. Zupo S, Massara R, Dono M et al. Apoptosis or plasma cell differentiation of CD38-positive B-chronic lymphocytic leukemia cells induced by crosslinking of surface IgM or IgD. Blood 2000;95(4):1.199-206.

28. Chen L, Widhopf G, Huynh L et al. Expression of ZAP-70 is associated with increased B-cell receptor signaling in chronic lymphocytic leukemia. Blood 2002;100(13):4.609-4.614.

29. Lanham S, Hamblin T, Oscier D et al. Differential signaling via surface IgM is associated with $\mathrm{VH}$ gene mutational status and CD38 expression in chronic lymphocytic leukemia. Blood 2003;101(3):1.087-1.093.

30. Deaglio S, Capobianco A, Bergui L et al. CD38 is a signaling molecule in Bcell chronic lymphocytic leukemia cells. Blood 2003;102(6):2.146-2.155.

31. Dohner H, Stilgenbauer S, Benner A et al. Genomic aberrations and survival in chronic lymphocytic leukemia. N Engl J Med 2000;343(26):1.910-1.916.

32. Krober A, Seiler T, Benner A et al. V(H) mutation status, CD38 expression level, genomic aberrations, and survival in chronic lymphocytic leukemia. Blood 2002;100(4):1.410-1.416.

33. Hamblin TJ, Davis Z, Gardiner A et al. Unmutated Ig V(H) genes are associated with a more aggressive form of chronic lymphocytic leukemia. Blood 1999;94(6):1.848-1.854.

34. Rosenwald A, Alizadeh AA, Widhopf G et al. Relation of gene expression phenotype to immunoglobulin mutation genotype in B cell chronic lymphocytic leukemia. J Exp Med 2001;194(11):1.639-1.647.

35. Wiestner A, Rosenwald A, Barry TS et al. ZAP-70 expression identifies a chronic lymphocytic leukemia subtype with unmutated immunoglobulin genes, inferior clinical outcome, and distinct gene expression profile. Blood 2003;101(12):4.944-4.951.

36. Vasconcelos Y, Davi F, Levy V et al. Binet's staging system and VH genes are independent but complementary prognostic indicators in chronic lymphocytic leukemia. J Clin Oncol 2003; 21:3.928-3.932.

Avaliação: Carlos Sergio Chiattone

(publicado após acordo do Editor)

Conflito de interesse: Artigo derivado do II Encontro Brasileiro de

Consenso da LLC

Recebido: 30/10/2005

Aceito: $15 / 11 / 2005$ 